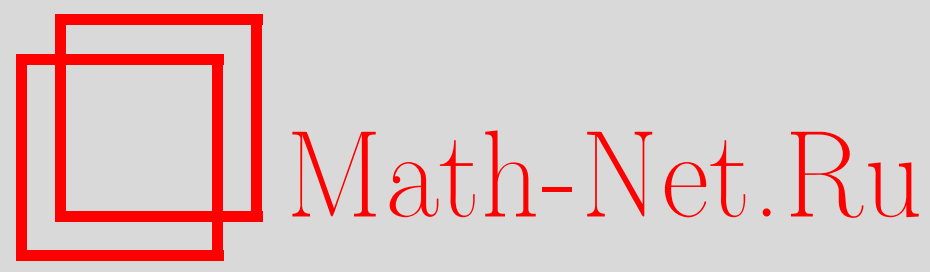

Л. А. Бекларян, Об аналогах альтернативы Титса для групп гомеоморфизмов окружности и прямой, Матем. заметки, 2002, том 71, выпуск 3, 334-347

DOI: https://doi.org/10.4213/mzm350

Использование Общероссийского математического портала Math-Net.Ru подразумевает, что вы прочитали и согласны с пользовательским соглашением http://www . mathnet.ru/rus/agreement

Параметры загрузки:

IP: 54.84 .234 .179

26 апреля 2023 г., 15:21:22

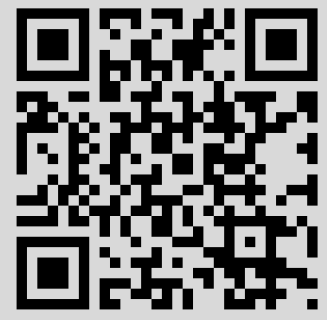




\section{ОБ АНАЛОГАХ АЛЬТЕРНАТИВЫ ТИТСА ДЛЯ ГРУПП ГОМЕОМОРФИЗМОВ ОКРУЖНОСТИ И ПРЯМОЙ}

\section{Л. А. Бекларян}

Г. Маргулисом была доказана гипотеза Гиза о справедливости аналога альтернативы Титса: для группы $G \subseteq \operatorname{Homeo}\left(S^{1}\right)$ гомеоморфизмов окружности или существует свободная подгруппа с двумя образующими, или существует инвариантная вероятностная мера на $S^{1}$. В этой заметке мы усиливаем результат Маргулиса: для группы $G \subseteq \operatorname{Homeo}\left(S^{1}\right)$ инвариантная вероятностная мера существует тогда и только тогда, когда факторгруппа $G / H_{G}$ не содержит свободной подгруппы с двумя образующими (здесь $H_{G}$ - некоторая каноническим образом определяемая подгруппа группы $G$ ). Сформулированы и доказаны аналоги альтернативы Титса для групп $G \subseteq \operatorname{Homeo}(\mathbb{R})$ гомеоморфизмов прямой.

Библиограффия: 7 названий.

Введение. В своем докладе на симпозиуме по динамическим системам, проходившем в Париже в июне 1998 года, Э. Гиз высказал предположение о справедливости для групп $G \subseteq \operatorname{Homeo}\left(S^{1}\right)$ аналога знаменитой альтернативы Титса: конечно-порожденная линейная группа либо содержит свободную подгруппу с двумя образующими, либо является почти разрешимой. В [1] Маргулис доказал гипотезу Гиза: для групшы $G \subseteq$ $\operatorname{Homeo}\left(S^{1}\right)$ или существует свободная подгруппа с двумя образующими, или существует инвариантная вероятностная мера на $S^{1}$. В этой заметке мы в качестве следствия из теоремы 6.1 в [2] формулируем следующий критерий существования инвариантной меры (теорема A): для групшы $G \subseteq \operatorname{Homeo}\left(S^{1}\right)$ инвариантная вероятностная мера существует тогда и только тогда, когда факторгрупа $G / H_{G}$ не содержит свободной подгруппы с двумя образующими (здесь $H_{G}$ - некоторая каноническим образом определяемая подгруппа группы $G$ ). Очевидно, такой критерий является усилением результата Маргулиса в [1]. В действительности, приводимый критерий допускает эквивалентную переформулировку в виде аналога альтернативы Титса (теорема $\mathrm{A}^{\prime}$ ): для групшы $G \subseteq \operatorname{Homeo}\left(S^{1}\right)$ факторгруппа $G / H_{G}$ либо содержит свободную подгруппу с двумя образуюшими, либо содержит коммутативную подгруппу индекса не более двух.

Далее, приводится критерий существования инвариантной меры для подгрупп группы $\operatorname{Homeo}(\mathbb{R})$ всех гомеоморфизмов прямой (теорема В): для групшы $G \subseteq \operatorname{Homeo}(\mathbb{R})$

Работа выполнена при поддержке Государственной программы поддержки научных школ, грант № 96-15-96135. 
инвариантная борелевская мера, конечная на компактах, существует тог да и только тогда, ког да минимальное множество непусто, а факторгруппа $G / H_{G}$ не содержит свободной подполугрупшы с двумя образуюшими. В действительности, приводимый критерий также допускает эквивалентную переформулировку в виде аналога альтернативы Титса (теорема $\mathrm{B}^{\prime}$ ): для групшы $G \subseteq \operatorname{Homeo}(\mathbb{R})$ с непустым минимальньм множеством факторгруппа $G / H_{G}$ либо содержит свободную подполугрупшу с двумя образующими, либо содержит коммутативную подгрупу индекса не более двух.

Отмеченная вьше теорема 6.1 из [2] позволяет получить критерий существования проективно-инвариантной меры для подгрупп группы $\operatorname{Homeo}(\mathbb{R})$ (теорема $\mathrm{C})$ : для группы $G \subseteq \operatorname{Homeo}(\mathbb{R})$, содержащей нормальную подгруппу $\Gamma \subseteq \operatorname{Homeo+}_{+}(\mathbb{R})$ с инвариантной мерой и свободно действующим элементом, проективно-инвариантная борелевская мера, конечная на компактах, существует тог да и только тогда, когда факторгруппа $G / H_{G}$ не содержит свободной подгрупшы с двумя образующими. В действительности, приводимый критерий также допускает эквивалентную переформулировку в виде аналога альтернативы Титса (теорема $\mathrm{C}^{\prime}$ ): для группы $G \subseteq \operatorname{Homeo}(\mathbb{R})$, содержащей нормальную подгруппу $\Gamma \subseteq \operatorname{Homeo}_{+}(\mathbb{R})$ с инвариантной мерой и свободно действующим элементом, факторгрупп $G / H_{G}$ либо содержит свободную подгруппу с двумя образуюшими, либо содержит разрешимую подгрупу ступени и индекса не более двух.

В. Солодовым в [3] для групш $G \subseteq$ Homeо+ $\left(S^{1}\right)$ получен результат, эквивалентньй альтернативе из [1], а в [4] для специальных конечно-порожденных групп $G \subseteq$ Homeо+ $_{+}(\mathbb{R})$ получен результат, эквивалентньй следующей, более слабой, альтернативе: или группа $G$ не содержит свободной подполугрупшы с двумя образуюшими, или сушествует инвариантная борелевская мера, конечная на компактах.

1. Критерий существования инвариантной меры для групп гомеоморфизмов окружности. Определение подгруппы $H_{G}$ связано с минимальными множествами для группы $G$, поэтому вначале приведем основные определения и факты, относящиеся к этим понятиям.

В дальнейшем $X$ обозначает либо $\mathbb{R}$, либо $S^{1}$. Пусть

$\operatorname{Homeo}(X)$ - группа всех гомеоморфизмов $X$, а Homeo+ $(X)$ - нормальная подгруппа сохраняюших ориентацию гомеоморфизмов;

Нотео $\left(S^{1}\right)$ - группа всех гомеоморфизмов прямой, являющихся накрытиями го-

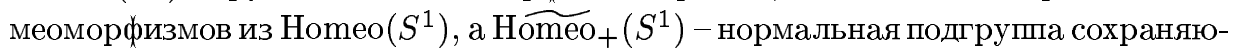
щих ориентацию гомеоморфизмов.

Наряду с группой $G \subseteq \operatorname{Homeo}(X)$ будем рассматривать ее нормальную подгруппу

$$
G_{+}=G \cap \mathrm{Homeo}_{+}(X)
$$

объединение стабилизаторов точек $X$, принадлежащих подгрупше $G_{+}$,

$$
G^{s}=\left\{g: g \in G_{+}, \exists t \in X g(t)=t\right\}
$$

и подмножества

$$
\begin{gathered}
G_{\infty}^{s}=\left\{g: g \in G^{s}, \inf \{t: g(t)=t\}=-\infty, \sup \{t: g(t)=t\}=+\infty\right\} \\
C=\left(G \backslash G^{s}\right) \cup G_{\infty}^{s}
\end{gathered}
$$


в случае $X=\mathbb{R}$, которые не всегда являются подгруппами. Заметим, что нормальная подгрупп $G_{+}$индекса не более двух. Для произвольного множества гомеоморфизмов $Q$ через $\langle Q\rangle$ будем обозначать группу, порожденную этими гомеоморфизмами, а также определим топологическую характеристику в виде множества неподвижных точек

$$
\text { Fix } Q=\{t: t \in X, \forall q \in Q q(t)=t\}
$$

Гомеоморфизм $g$ называется свободно действующим, если $\mathrm{Fix}\langle g\rangle=\varnothing$.

ОПредЕЛЕНИЕ 1. Пусть $G \subseteq \operatorname{Homeo}(X)$. Непустое подмножество $X$ называется минимальным, если оно замкнуто, $G$-инвариантно и не содержит собственных замкнутых $G$-инвариантных подмножеств. Если минимальное множество единственное, то его будем обозначать через $E(G)$. Если не существует минимального множества, то по определению будем полагать, что оно пустое множество.

Оказьвается, что для груп, действующих на окружности, а также групп $G \subseteq$ Нотео $+\left(S^{1}\right)$, минимальное множество всегда не пусто, и, кроме того, существуют жесткие ограничения на его топологическую структуру как следствие одномерности фазового пространства.

Теорема 1. Пусть $G \subseteq \mathrm{HomeO+}_{+}(X)$. Тогда справедливо в точности одно из следующих утверәдений:

а) любое минимальное мноэество дискретно и содерэится в $\mathrm{Fix} G^{s}, a \mathrm{Fix} G^{s}$ состоит из оббединения дискретных минимальных множеств;

б) минимальное мнохество единственно, является совершенным нигде не плотным подмножеством $X$ и содержится в замыкании орбиты $\overline{G(t)}$ произвольной точки $t \in X$;

в) минимальное мнохсество совпадает с $X$;

г) минимальное мнохсество пустое (в случае $\left.G \subseteq \mathrm{Homeo}_{+}(\mathbb{R})\right)$.

ДокАЗАтЕльство. Случай $X=\mathbb{R}$ рассмотрен в работе [5]. Рассмотрим случай окружности $X=S^{1}$. Для элемента $g \in G$ через $l_{g}$ обозначим гомеоморфизм прямой, являющийся накрытием гомеоморфизма окружности $g$ и нормированньй условием $0 \leqslant l_{g}(0)<1$. Образуем групу $\widehat{G}=\left\langle\left\{l_{g}\right\}_{g \in G}, \bar{g}\right\rangle$, где $\bar{g}(t)=t+1$. Пусть $\lambda: \mathbb{R} \rightarrow S^{1}-$ отображение вида $\lambda(t)=t-[t]$, где $[\cdot]$ означает целую часть числа. Такое отображение индуцирует естественньй эпиморфизм $\pi: \widehat{G} \rightarrow G$ с ядром $\operatorname{ker} \pi=\langle\bar{g}\rangle$. Заметим, что при отображении $\lambda$ образ минимального множества группы $\widehat{G}$ совпадает с минимальным множеством группы $G$. В таком случае доказательство следует из справедливости теоремы для группы $\widehat{G}$.

ПРЕДЛОЖЕНИЕ 1. Пусть $G \subseteq \operatorname{Homeо}(X)$. Минимальные множества группы $G$ и нормальной подгруппы $G_{+}$непусты одновременно. Каждое минимальное множество группы $G$ состоит из обгединения не более чем двух минималных множеств нормальной подгруппы $G_{+}$.

ДокАЗАтЕльство. Следует рассмотреть случай $G \neq G_{+}$. Мы знаем, что $G=$ $G_{+} \cup f G_{+}$, где $f-$ некоторьй элемент из $G \backslash G_{+}$. Поэтому если множество $B$ инвариантно относительно подгрупшы $G_{+}$, то множество $f(B)$ также будет инвариатным 
относительно подгруппы $G_{+}$. Более того, так как $f^{2}(B)=B$, то множество $B \cup f(B)$ будет инвариантным относительно самой групы $G$.

Пусть для подгруппы $G_{+}$существует непустое минимальное множество $U$. Как уже отмечалось вьше, множество $f(U)$ инвариантно относительно подгрупшы $G_{+}$, а множество $U \cup f(U)$ будет инвариантным относительно самой группы $G$. Следовательно, для группы $G$ множество $U \cup f(U)$ является минимальньм.

Пусть минимальное множество групшы $G$ непусто и $D$ является таким минимальньм множеством. В силу инвариантности для любого $g \in G$ его действие на минимальном множестве является гомеоморфизмом. Предположим, что для подгрупшы $G_{+}$не сушествует минимального множества. Тогда существует последовательность вложенных замкнутых подмножеств $D_{n}, n=0,1,2, \ldots, D_{0}=D$, которые инвариантны относительно нормальной подгрупшы $G_{+}$и удовлетворяют условию $\bigcap_{n=0}^{\infty} D_{n}=\varnothing$. Выберем точку $\bar{t} \in D$. Найдется натуральное число $\bar{n}$ такое, что точки $\bar{t}, f^{-1}(\bar{t})$ не будут принадлежать множеству $D_{\bar{n}}$. В таком случае точка $\bar{t}$ не будет принадлежать замьканию множества $D_{\bar{n}} \cup f\left(D_{\bar{n}}\right)$, и оно будет собственным замкнутым подмножеством множества $D$, инвариантным относительно групшы $G$. Это противоречит условию минимальности множества $D$, откуда и следует непустота минимального множества для подгрупшы $G_{+}$.

И последнее. Если минимальное множество подгруппы $G_{+}$недискретное, то по теореме 1 оно единственное и обозначается через $E\left(G_{+}\right)$. Из инвариантности множества $f\left(E\left(G_{+}\right)\right)$относительно подгрупшы $G_{+}$и теоремы 1 следует включение $E\left(G_{+}\right) \subseteq$ $f\left(E\left(G_{+}\right)\right)$. Из полученного включения следует, что $f\left(E\left(G_{+}\right)\right) \subseteq f^{2}\left(E\left(G_{+}\right)\right)$. С другой стороны, из инвариантности множества $E\left(G_{+}\right)$относительно подгруппы $G_{+}$и условия $f^{2} \in G_{+}$следует, что $f^{2}\left(E\left(G_{+}\right)\right)=E\left(G_{+}\right)$. Тогда из полученных включений следует, что $f\left(E\left(G_{+}\right)\right)=E\left(G_{+}\right)$, т.е. минимальные множества групшы $G$ и ее нормальной подгрупшы $G_{+}$совпадают.

Если $U$ - дискретное минимальное множество подгрупшы $G_{+}$, то множество $f(U)$ также будет дискретным минимальным множеством для подгрупшы $G_{+} \cdot$ Множества $U$ и $f(U)$ либо не пересекаются, либо совпадают, а множество $U \cup f(U)$ является минимальным множеством для исходной группы $G$ и состоит из не более чем двух минимальных множеств подгруппы $G_{+}$.

ОпРЕДЕЛЕниЕ 2. Для групшы $G \subseteq \operatorname{Homeo}(X)$ определим нормальную подгруппу $H_{G}$ следуюшим образом:

1) если минимальное множество непусто и недискретно, то положим

$$
H_{G}=\left\{h: h \in G_{+}, E(G) \subseteq \operatorname{Fix}\langle h\rangle\right\}
$$

2) если минимальное множество непусто и дискретно, то положим $H_{G}=G^{s}$ (по теореме 1 из дискретности минимального множества следует непустота множества Fix $G^{s}$; из непустоты Fix $G^{s}$ следует, что $G^{s}$ является групой, а из инвариантности множества Fix $G^{s}$ следует нормальность подгрупшы $G^{s}$ );

3) если минимальное множество пусто,то положим $H_{G}=\langle e\rangle$.

ОПРЕДЕЛЕНИЕ 3. Борелевская мера $\mu$, конечная на компактах, назьвается проективно-инвариантной относительно группы $G \subseteq \operatorname{Homeo}(\mathbb{R})$, если для любого $g \in G$ 
существует число $c(g)>0$ такое, что для всякого борелевского множества $B$ выполняется условие $\mu\left(g^{-1}(B)\right)=c(g) \mu(B)$.

Если для любого $g \in G c(g)=1$, то такая проективно-инвариантная мера является инвариантной. В дальнейшем, говоря о мере, мы будем подразумевать, что это борелевская мера, конечная на компактах. В случае окружности мы будем предполагать, что это вероятностная мера.

Приведем наиболее важньй топологический критерий существования инвариантной меры.

Теорема 2. Для группы $G \subseteq \mathrm{HomeO}_{+}(X)$ инвариантная мера существует тогда и только тогда, когда множество Fix $G^{s}$ непусто.

ДокАЗАтельство. Случай $X=\mathbb{R}$ рассмотрен в работе [6]. Рассмотрим случай окружности $X=S^{1}$. Пусть групша $\widehat{G}$ и отображения $\bar{g}, \lambda, \pi$ те же, что и при доказательстве теоремы 1 . Несложно заметить, что множества Fix $\widehat{G}^{s}$ и Fix $G^{s}$ непусты одновременно и при отображении $\lambda$ образ множества Fix $\widehat{G}^{s}$ совпадает с множеством Fix $G^{s}$. С другой стороны, для групшы $\widehat{G}$ инвариантная мера существует тогда и только тогда, когда для группы $G$ сушествует инвариантная вероятностная мера. Тогда утверждение теоремы в случае окружности $S^{1}$ будет следовать из утверждения теоремы для случая $X=\mathbb{R}$, примененного к группе $\widehat{G}$.

В работе [6] были изучены свойства инвариантных мер и, в частности, их носители.

Теорема 3. Пусть $G \subseteq \mathrm{HomeO}_{+}(X)$. Тогда носитель supp $\mu$ инвариантной меры $\mu$ состоит из некоторого оббединения минимальных множсеств. Если минимальное мнохество недискретно, а $\mu_{1} u \mu_{2}-$ инвариантные меры, то $\mu_{2}=d \mu_{1}$, əде $d>0$.

ДоказАтельство. Случай $X=\mathbb{R}$ рассмотрен в работе [6]. Рассмотрим случай окружности $X=S^{1}$. Пусть групша $\widehat{G}$ и отображения $\bar{g}, \lambda, \pi$ те же, что и при доказательстве теоремы 1 . Заметим, что для групшы $\widehat{G}$ инвариантная мера существует тогда и только тогда, когда для группы $G$ существует инвариантная вероятностная мера. С другой стороны, минимальное множество группы $G$ недискретно тогда и только тогда, когда недискретно минимальное множество. Тогда утверждение теоремы в случае окружности $S^{1}$ будет следовать из утверждения теоремы для случая $X=\mathbb{R}$, примененного к группе $\widehat{G}$.

ПРЕДЛОЖЕНИЕ 2. Пусть $G \subseteq \operatorname{Homeo}(X)$. Для группь $G$ инвариантная мера существует тогда и только тогда, когда существует инвариантная мера для нормальной подгруппь $G_{+}$.

ДоКАЗАТЕЛЬСтвО. В одну сторону доказательство очевидно. Пусть для нормальной подгрупшы $G_{+}$сушествует инвариантная мера $\mu$. Следует рассмотреть случай $G \neq$ $G_{+}$. Мы знаем, что $G=G_{+} \cup f G_{+}$, где $f$ - некоторьй элемент из $G \backslash G_{+}$. Пусть минимальное множество подгруппы $G_{+}$дискретное. Обозначим его через $D$. Тогда дискретное множество $f(D)$ также является минимальным множеством подгруппы $G_{+}$и по предложению 1 объединение $D \cup f(D)$ будет дискретным минимальным множеством 
самой групшы $G$. Имея дискретное минимальное множество групшы $G$, очевидным образом строится мера, инвариантная относительно $G$. Пусть минимальное множество нормальной подгрупшы $G_{+}$недискретное. Если $q$ - гомеоморфизм, то определим меру $q_{*} \mu$ по следуюшему правилу: для любого борелевского множества $B$ должно вьполняться условие $q_{*} \mu(B)=\mu\left(q^{-1}(B)\right)$. Из нормальности подгрупшы $G_{+}$следует, что для любого элемента $g \in G$ мера $g_{*} \mu$ также инвариантна относительно подгрупшы $G_{+} \cdot$ Так как минимальное множество подгрупшы $G_{+}$недискретное, то по теореме $3 g_{*} \mu=d_{g} \mu$, где $d_{g}>0$. Очевидно, что для элементов $g \in G_{+}$значение $d_{g}$ равно единице. Для элемента $g \neq G_{+}$справедливо условие $g^{2} \in G_{+}$, поэтому должно вьполняться равенство $d_{g}^{2}=1$. Следовательно, для любого элемента $g \in G$ также должно вьполняться условие $d_{g}=1$, откуда и следует инвариантность меры $\mu$ относительно исходной группы $G$.

Сформулируем одно утверждение, непосредственно вытекающее из следствия 7.3 работы [5].

ПРЕДЛОЖЕНИЕ 3. Пусть $G \subseteq \widetilde{\text { НотеО}}_{+}\left(S^{1}\right)$. ДЛя группь $G$ проективно-инвариантная мера существует тогда и только тогда, когда существует инвариантная мера.

ОПРЕДЕЛЕНИЕ 4. Пусть $G \subseteq \operatorname{Homeo}_{+}(\mathbb{R})$. Нормальная подгруппа $M \subseteq G$ называется 0-максимальной, если существует борелевская мера, конечная на компактах и инвариантная относительно подгрупшы $M$, и $M$ не является собственной подгрупшой какой-либо нормальной подгруппы группы $G$, для которой также существует инвариантная борелевская мера, конечная на компактах.

В [2] было показано, что для групп $G \subseteq \mathrm{Homeo+}_{+}(\mathbb{R})$ с непустьм минимальным множеством существует 0-максимальная подгруппа $M$ и справедливо включение $H_{G} \subseteq M$.

Приведем формулировку теоремы 6.1 из работы [2].

TEOPEMA 4. Пусть $G \subseteq \mathrm{HomeO}_{+}(\mathbb{R})$, для которой существует непустое минимальное множество и 0-максимальная подгруппа $M$ со свойством $M \neq H_{G}$. Тогда для существования борелевской меры, конечной на компактах и проективно-инвариантной относительно группы $G$, необходимо и достаточно, чтобы факторгруппа $G / H_{G}$ не содержала свободной подгруппы с двумя образующими.

Для групा $G \subseteq$ Нотео $_{+}\left(S^{1}\right)$ теорема 4 может быть уточнена.

ТеОрема 5. Пусть $G \subseteq$ Нотео+ $\left(S^{1}\right)$. Тогда для существования борелевской меры, конечной на компактах и инвариантной относительно группы $G$, необходимо и достаточно, чтобы факторгруппа $G / H_{G}$ не содерхала свободной подгруппы $c$ двумя образующими.

ДокАЗАТЕЛЬСТво. По предложению 3 для групшы $G$ проективно-инвариантная мера существует тогда и только тогда, когда существует инвариантная мера. Образуем группу $\Pi=\langle G, \bar{g}\rangle$, где $\bar{g}(t)=t+1$. Для произвольной групшы $Q \subseteq \mathrm{Homeo+}_{+}\left(S^{1}\right)($ см. [5]) минимальное множество непусто, а инвариантная мера существует тогда и только тогда, когда существует инвариантная мера для группы $\langle Q, \bar{g}\rangle$. Отсюда следует, что для группы П 0-максимальная подгрупша $M$ существует и содержит элемент $\bar{g}$. Поэтому 


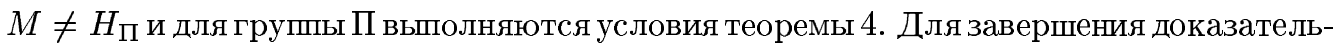
ства остается заметить, что факторгрупшы $G / H_{G}$ и П $/ H_{\Pi}$ одновременно либо содержат свободную подгруппу с двумя образующими, либо ее не содержат.

Теперь мы можем сформулировать и доказать наш основной результат для групп, действующих на окружности.

Теорема А. Пусть $G \subseteq \operatorname{Homeo}\left(S^{1}\right)$. Для существования вероятностной ме$p$ и, инвариантной относительно группь $G$, необходимо и достаточно, чтобы факторгруппа $G / H_{G}$ не содержала свободной подгруппы с двумя образующими.

ДокАЗАТЕЛЬСтво. Заметим, что $H_{G}=H_{G_{+}}$. По предложению 2 для группы $G$ и ее нормальной подгрупы $G_{+}$инвариантная мера либо существует, либо нет одновременно. С другой стороны, для факторгрупा $G / H_{G}, G_{+} / H_{G}$ свободные подгрупшы с двумя образующими либо существуют, либо нет также одновременно. Поэтому нам достаточно доказать теорему для групп $G \subseteq$ Homeo $_{+}\left(S^{1}\right)$. Для элемента $g \in G$ через $l_{g}$ обозначим гомеоморфизм прямой, являющийся накрытием гомеоморфизма окружности $g$ и нормированный условием $0 \leqslant l_{g}(0)<1$. Образуем группу $\widehat{G}=\left\langle\left\{l_{g}\right\}_{g \in G}, \bar{g}\right\rangle$, где $\bar{g}(t)=t+1$. Для группы $G$ инвариантная мера существует тогда и только тогда, когда она существует для групшы $\widehat{G}$. Пусть $\Gamma=\left\langle H_{\widehat{G}}, \bar{g}\right\rangle$. Ядро естественного эпиморфизма $\pi: \widehat{G} \rightarrow G$ равно $\operatorname{ker} \pi=\langle\bar{g}\rangle$ и $\pi(\Gamma)=H_{G}$. Следовательно, факторгрупшы $\widehat{G} / \Gamma$ и $G / H_{G}$ изоморфны. С другой стороны, факторгруппы $\widehat{G} / \Gamma$ и $\widehat{G} / H_{\widehat{G}}$ одновременно либо содержат свободную подгруппу с двумя образующими, либо ее не содержат. В таком случае для групшы $\widehat{G}$ вьполняются все условия теоремы 5 , откуда и следует доказательство.

ЗАмечАние 1. Для группы $G \subseteq \operatorname{Homeo+}_{+}(X)$ факторгруппа $G / H_{G}$ может быть реализована как грушпа действий на $X$ (см. [6]). Для любого класса смежности $g H_{G}$ соответствующее ей действие на минимальном множестве совпадает с действием элемента $g$, а вне минимального множества действует растяжениями на максимальных открытых интервалах. При этом инвариантная мера для групшы $G$ является инвариантной мерой для факторгруппы $G / H_{G}$, и наоборот.

ЗАмечание 2. Для групп $G \subseteq$ Нотео+ $_{+}\left(S^{1}\right)$ в работе В. Солодова [3] сформулирована альтернатива, эквивалентная альтернативе из [1], в которой условие существования инвариантной меры заменено на эквивалентное ей условие непустоты множества $\mathrm{Fix} G^{s}$. Эквивалентность этих двух условий устанавливается теоремой 2.

Теорема А допускает эквивалентную переформулировку в виде аналога альтернативы Титса. Для этого приведем теорему о факторгрупше.

Teорема 6. Пусть $G \subseteq$ Homeo+ $_{+}(X)$. Тогда факторгруппа $G /\left\langle G^{s}\right\rangle$ коммутативна и изоморфна подгруппе группы $X$.

ДокАЗАтЕльство. В случае $X=\mathbb{R}$ доказательство приведено в [7]. Рассмотрим случай окружности $X=S^{1}$. Пусть груша $\widehat{G}$ и отображения $\bar{g}, \lambda, \pi$ те же, что и при доказательстве теоремы 1. Очевидно, что $\pi(\mathscr{T})=\left\langle G^{s}\right\rangle$, где $\mathscr{T}=\left\langle\widehat{G}^{s}, \bar{g}\right\rangle$. В таком случае существуют эпиморфизм $\widehat{G} /\left\langle\widehat{G}^{s}\right\rangle \rightarrow \widehat{G} / \mathscr{T}$ и изоморфизм $\widehat{G} / \mathscr{T} \simeq G /\left\langle G^{s}\right\rangle$. Так как факторгруппа $\widehat{G} /\left\langle\widehat{G}^{s}\right\rangle$ изоморфна подгрупше адлитивной группы $\mathbb{R}$, то факторгрупша $G / G^{s}$ будет изоморфна подгруппе группы $S^{1}$. 
Теперь мы можем сфформулировать и доказать аналог альтернативы Титса для групп, действующих на окружности.

Лемма 1. Для группы $G \subseteq$ Homeo+ $_{+}\left(S^{1}\right)$ факторгрупnа $G / H_{G}$ либо содержит свободную подгруппу с двумя образующими, либо является коммутативной.

ДокАЗАТЕЛЬСтво. Пусть $G / H_{G}$ не содержит свободной подгруппы с двумя образующими. По теореме А для группы $G$ существует инвариантная вероятностная мера, а по теореме 2 множество $\mathrm{Fix} G^{s}$ непусто. Из непустоты множества Fix $G^{s}$ следует, что множество $G^{s}$ образует группу, т.е. $\left\langle G^{s}\right\rangle=G^{s}$. В силу инвариантности множества $\mathrm{Fix} G^{s}$ минимальное множество будет принадлежать Fix $G^{s}$. Если минимальное множество дискретное, то по определению $H_{G}=G^{s}$. Если минимальное множество недискретное, то в силу определения множества $\mathrm{Fix} G^{s}$ также справедливо условие $H_{G}=G^{s}$. В таком случае коммутативность факторгрупшы $G /\left\langle H_{G}\right\rangle$ будет следовать из теоремы 6 . Обратное утверждение очевидно.

TеOрема А'. Для групnы $G \subseteq \operatorname{Homeo}\left(S^{1}\right)$ факторгрупnа $G / H_{G}$ либо содерэит свободную подгруппу с двумя образующими, либо является разрешимой ступени не более двух (содержит коммутативную подгруппу индекса не более двух).

ДокАЗАтЕльство. Заметим, что $H_{G}=H_{G_{+}}$. Для факторгрупп $G / H_{G}, G_{+} / H_{G}$ свободные подгрупшы с двумя образуюшими либо существуют, либо нет одновременно. Если факторгруппа $G_{+} / H_{G}$ не содержит свободной подгруппы с двумя образующими, то по лемме 1 она является коммутативной. В таком случае факторгруппа $G / H_{G}$ будет содержать коммутативную подгруппу индекса не более двух.

2. Критерий существования инвариантной меры для групп гомеоморфизмов прямой. Для исследования групп, действующих на прямой, приведем один признак непустоты множества $\mathrm{Fix} G^{s}$, которьй является непосредственным следствием теоремы 8.4 из [5].

ПРЕДЛОЖЕНИЕ 4. Пусть $G \subseteq \mathrm{HomeO}_{+}(\mathbb{R})$. Ecли группа $G$ не содержит свободной подполугруппы с двумя образующими $u G \backslash G_{\infty}^{s} \neq \varnothing$, то множество Fix $G^{s}$ неnyсmo.

Теперь мы можем сформулировать наш основной результат для груп, действующих на прямой.

Теорема В. Пусть $G \subseteq \operatorname{Homeo}(\mathbb{R})$. Для существования борелевской мерь, конечной на компактах и инвариантной относительно группы $G$, необходимо и достаточно, чтобы выполнялись условия:

1) для группы $G$ существует непустое минимальное множсество;

2) факторгруппа $G / H_{G}$ не содержит свободной подполугруппьи с двумя образующими.

ДокАЗАТЕЛЬСтво. Заметим,что $H_{G}=H_{G_{+}}$, а минимальные множества групшы $G$ и подгруппы $G_{+}$непусты одновременно (предложение 1). По предложению 2 для группы $G$ и ее нормальной подгрупшы $G_{+}$инвариантная мера либо существует, либо нет 
одновременно. С другой стороны, для факторгрупп $G / H_{G}, G_{+} / H_{G}$ свободные подполугруппы с двумя образующими либо существуют, либо нет также одновременно. В таком случае доказательство теоремы достаточно провести для групा $G \subseteq \mathrm{Homeo}_{+}(\mathbb{R})$.

Необходимость. Пусть существует $\sigma$-конечная борелевская мера $\mu$, инвариантная относительно группы $G$. Тогда по теореме $2 \mathrm{Fix}^{s} \neq \varnothing$. Из непустоты множества $\mathrm{Fix} G^{s}$ и его инвариантности следует, что минимальное множество непусто и содержится в Fix $G^{s}$. С другой стороны, из условия Fix $G^{s} \neq \varnothing$ следует, что $G^{s}$ является группй, т.е. $\left\langle G^{s}\right\rangle=G^{s}$. Если минимальное множество дискретное, то по определению $H_{G}=G^{s}$ и в силу теоремы 6 факторгруппа $G / H_{G}$ будет коммутативной группой, а потому не может содержать свободных подполугрупп с двумя образующими. Если минимальное множество недискретное, то в силу определения множества Fix $G^{s}$ так же, как и в предыдущем случае, $H_{G}=G^{s}$ и по теореме 6 факторгруппа $G / H_{G}$ будет коммутативной. Необходимость доказана.

Достаточность. Если минимальное множество дискретное, то по теореме 1 оно содержится в множестве $\operatorname{Fix} G^{s}$ и поэтому $\operatorname{Fix} G^{s} \neq \varnothing$. Из условия $\operatorname{Fix} G^{s} \neq \varnothing$ и теоремы 2 следует существование меры, инвариантной относительно группы $G$, что и требовалось доказать.

Пусть минимальное множество недискретное и в силу теоремы 1 оно единственное (обозначается через $E(G)$ ). Если $G \backslash G_{\infty}^{s} \neq \varnothing$, то из условия 2 ) и предложения 4 следует, что для группы $G$ вьполняется свойство $\mathrm{Fix} G^{s} \neq \varnothing$. Тогда по теореме 2 существует инвариантная мера.

Остается рассмотреть случай, когда $G=G_{\infty}^{s}$. Покажем, что для любого элемента $g \in G$ и любой точки $t \in E(G)$ вьполняется условие $g(t)=t$, т.е. точка $t$ является неподвижной для элемента $g$. Действительно, пусть для некоторой точки $\bar{t} \in E(G)$ и некоторого элемента $g_{1} \in G$ выполняется условие $g_{1}(\bar{t}) \neq \bar{t}$. Заметим, что такой элемент $g_{1}$ не принадлежит подгрупе $H_{G}$. Для определенности будем полагать, что $g_{1}(\bar{t})>\bar{t}$. Определим точки

$$
t_{1}=\sup \left\{t: g_{1}(t)=t, t<\bar{t}\right\}, \quad t_{2}=\inf \left\{t: g_{1}(t)=t, t>\bar{t}\right\}
$$

Так как $G=G_{\infty}^{s}$ и $g_{1}(\bar{t})>\bar{t}$, то $t_{1}, t_{2}$ конечны и удовлетворяют условию $t_{1}<\bar{t}<t_{2}$. Из условия $\bar{t} \in E(G)$ в силу свойства б) теоремы 1 следует, что точка $\bar{t}$ принадлежит замыканию орбиты $G\left(t_{1}\right)$. В таком случае найдутся элемент $g_{2} \in G$ и натуральное число $k$, для которых справедливы условия $g_{1}^{-k} g_{2}\left(t_{1}\right)>t_{1}, t_{1}<\tilde{t}<\bar{t}$, где $\tilde{t}=\inf \left\{t: g_{1}^{-k} g_{2}(t)=t\right.$, $\left.t>t_{1}\right\}$. Заметим, что элемент $g_{1}^{-k} g_{2}$ также не принадлежит подгруппе $H_{G}$. Рассмотрим интервал $\left[t_{1}, \widetilde{t}\right]$. Для элемента $g_{1}$ выполняется условие $g_{1}\left(t_{1}\right)=t_{1}$, а на полуинтервале $\left.] t_{1}, \widetilde{t}\right]$ нет неподвижных точек. Для элемента $g_{1}^{-k} g_{2}$ вьполняется условие $g_{1}^{-k} g_{2}(\widetilde{t})=\widetilde{t}$, а на полуинтервале $\left[t_{1}, \widetilde{t}\right.$ нет неподвижных точек. В таком случае (см. [4, лемма 3.1]) групша $\left\langle g_{1}, g_{1}^{-k} g_{2}\right\rangle$ содержит свободную подполугруппу с двумя образующими. Так как элементы $g_{1}$ и $g_{1}^{-k} g_{2}$ не принадлежат нормальной подгруппе $H_{G}$, то отсюда следует, что факторгруппа $H_{G}$ содержит свободную подполугруппу с двумя образуюшими. Противоречие. Следовательно, для любых $g \in G$ и $t \in E(G)$ справедливо условие $g(t)=t$. Но это означает, что Fix $G^{s} \neq \varnothing$ и по теореме 2 существует мера, инвариантная относительно группы $G$. Теорема доказана. 
ЗАмЕчАниЕ 3. Для конечно-порожденных групп минимальное множество всегда непусто [5]. В конще введения сформулирована альтернатива, которая слабее, чем теорема В. Для специальных конечно-порожденных групп $G \subseteq$ Homeo+ $_{+}(\mathbb{R})$ в работе Солодова [4] доказывается эквивалентная альтернатива, в которой условие существования инвариантной меры заменено на условие непустоты множества Fix $G^{s}$. Эквивалентность этих двух условий устанавливается теоремой 2.

Теорема В допускает эквивалентную переформулировку в виде аналога альтернативы Титса.

ЛЕмма 2. Для группь $G \subseteq \mathrm{Homeo}_{+}(\mathbb{R})$ с непустым минимальным множеством факторгруппа $G / H_{G}$ либо содержит свободную подполугруппу с двумя образуюшими, либо является коммутативной.

ДокАЗАТЕЛЬСТво. Пусть $G / H_{G}$ не содержит свободной подполугруппы с двумя образуюшими. По теореме 4 для группы $G$ существует инвариантная мера, а по теореме 2 множество Fix $G^{s}$ непусто. Из непустоты множества Fix $G^{s}$ следует, что множество $G^{s}$ образует группу, т.е. $\left\langle G^{s}\right\rangle=G^{s}$. В силу инвариантности множества Fix $G^{s}$ минимальное множество будет принадлежать $\mathrm{Fix} G^{s}$. Если минимальное множество дискретное, то по определению $H_{G}=G^{s}$. Если минимальное множество недискретное, то в силу определения множества $\mathrm{Fix} G^{s}$ также справедливо условие $H_{G}=G^{s}$. В таком случае коммутативность факторгруппы $G /\left\langle H_{G}\right\rangle$ будет следовать из теоремы 6 . Обратное утверждение очевидно.

Теорема В'. Для групnы $G \subseteq \operatorname{Homeo}(\mathbb{R})$ с непустым минимальным множсеством факторгруппа $G / H_{G}$ либо содержит свободную подполугруппу с двумя образуюшими, либо является разрешимой ступени не более двух (содержит коммутативную подгруппу индекса не более двух).

ДокАЗАТЕЛЬСтво. Заметим, что $H_{G}=H_{G_{+}}$, а минимальные множества группы $G$ и подгруппы $G_{+}$непусты одновременно (предложение 1 ). Для факторгрупп $G / H_{G}$ и $G_{+} / H_{G}$ свободные подполугрупш с двумя образующими либо существуют, либо нет одновременно. Если факторгруппа $G_{+} / H_{G}$ не содержит свободной подгруппы с двумя образующими, то по лемме 2 она является коммутативной. В таком случае факторгруппа $G / H_{G}$ будет содержать коммутативную подгруппу индекса не более двух.

3. Критерий существования проективно-инвариантной меры для групп, действующих на прямой. Приведем одно необходимое условие пустоты минимального множества и одно достаточное условие существования инвариантной меры (см. [2, лемма 2.1, предложение 3.1$]$ ).

ТЕОрема 7. Пусть $G \subseteq$ Нотео+ $(\mathbb{R})$. Если минимальное множество пустое, $m o G=G_{\infty}^{s}$.

ПРЕДЛОЖЕНИЕ 5. Пусть $G \subseteq$ Hотео+ $_{+}(\mathbb{R})$. Если множсество $G^{s}$ образует подәруппу и $G \backslash G^{s} \neq \varnothing$, то существует инвариантная мера.

Сформулируем одно свойство групп с проективно-инвариантной мерой, описанное в теореме 7.4 из [5]. 
ПРЕДЛОЖЕНИЕ 6. Пусть $G \subseteq \mathrm{HomeO}_{+}(\mathbb{R})$, для которой существует проективно-инвариантная мера, но не существует инвариантной меры. Тогда для любых проективно-инвариантных мер $\mu_{1}, \mu_{2}$ справедливо условие $\mu_{2}=d \mu_{1}, d>0$.

ПРЕДЛОЖЕНИЕ 7. Пусть $G \subseteq \operatorname{Homeo}(\mathbb{R})$. Для группь $G$ проективно-инвариантная мера существует тогда и только тогда, когда существует проективно-инвариантная мера для нормальной подгруппь $G_{+}$.

ДокАЗАТЕЛЬСТво. В одну сторону доказательство очевидно. Пусть для нормальной подгруппы $G_{+}$существует проективно-инвариантная мера $\mu$. Если для $G_{+}$существует инвариантная мера, то по предложению 2 для группы $G$ также существует инвариантная мера. Остается рассмотреть случай, когда для $G_{+}$не существует инвариантной меры. Из нормальности подгруппы $G_{+}$следует, что для любого $g \in G$ мера $g_{*} \mu$ также будет проективно-инвариантной. Тогда по предложению 6 для любого $g \in G$ $g_{*} \mu=d_{g} \mu, d_{g}>0$, откуда и следует, что мера $\mu$ будет проективно-инвариантной относительно грушшы $G$.

Лемма 3. Пусть $G \subseteq \mathrm{HomeO}_{+}(\mathbb{R})$. Для существования непустого минимального множества и 0 -максимальной подгруппы $M$ со свойством $M \neq H_{G}$ необходимо и достаточно, чтобы существовала нормальная подгруппа Г с инвариантной мерой и свободно действующим әлементом.

ДокАЗАТЕЛЬСтво. Необходимость. Пусть минимальноемножество группы $G$ будет дискретным. Тогда по определению $H_{G}=G^{s}$, а по условию теоремы $M \neq G^{s}$, откуда и следует, что нормальная подгруппа $M$ содержит свободно действующий элемент. Остается выбрать подгруппу Г равной $M$.

Пусть минимальное множество группы $G$ недискретное и поэтому единственное. Так как для подгрупшы $M$ существует инвариантная мера $\mu$, то по теореме 2 замкнутое множество $\mathrm{Fix} M^{s}$, инвариантное относительно $M$, непусто. В силу теорем 1 и 3 носитель меры supp $\mu$ меры $\mu$, состояший из некоторого объединения минимальных множеств, будет принадлежать множеству $\mathrm{Fix} M^{s}$. В силу нормальности подгруппы $M$ для любого $g \in G$ мера $g_{*} \mu$ также будет инвариантной относительно подгрупшы $M$ и, следовательно, для нее также вьполняется включение supp $g_{*} \mu \subseteq \operatorname{Fix} M^{s}$. Заметим, что $\operatorname{supp} g_{*} \mu=g^{-1}(\operatorname{supp} \mu)$. Через $U$ обозначим замькание объединения $\bigcup_{g \in G} g(\operatorname{supp} \mu)$. Очевидно, что $U$ принадлежит замкнутому множеству Fix $M^{s}$ и является инвариантным относительно группы $G$. С другой стороны, по теореме 1 минимальное множество $E(G)$ будет принадлежать множеству $U$, откуда и следует включение $E(G) \subseteq \operatorname{Fix} M^{s}$. Из последнего включения следует, что $M^{s} \subseteq H_{G}$. Тогда из условия $M \neq H_{G}$ будет следовать, что $M \neq M^{s}$, т.е. в $M$ существует свободно действующий элемент. Остается положить Г равной $M$.

Достаточность. Так как в Г существует свободно действующий элемент, т.е. $\Gamma \neq \Gamma^{s}$, то по теореме 7 минимальное множество исходной группы $G$ непусто. Пусть минимальное множество групш $G$ дискретное. Тогда по теореме 1 множество Fix $G^{s}$ непусто, а по теореме 2 для группы $G$ существует инвариантная мера. С другой стороны, по определению для групп с дискретньм минимальным множеством имеет место условие $H_{G}=G^{s}$. Так как $\Gamma \neq \Gamma^{s}$, то и $G \neq G^{s}$. Остается положить $M$ равной исходной группе $G$.

Пусть минимальное множество группы $G$ недискретное, а потому единственное и обозначается $E(G)$. Для подгрупш $\Gamma$, повторяя все рассуждения, проведенные для под- 
группы $M$ во втором абзаце доказательства необходимости,получим включение $E(G) \subseteq$ Fix $\Gamma^{s}$. Из этого включения следует, что $\Gamma^{s} \subseteq H_{G}$. Образуем группу $\mathscr{F}=\left\langle\Gamma, H_{G}\right\rangle$. Так как $E(G)$ инвариантно относительно подгрупшы $\Gamma$, то для группы $\mathscr{F}$ будет вьполняться условие $\mathscr{F}^{s}=H_{G}$. В таком случае $E(G) \subseteq$ Fix $\mathscr{F}^{s}$ и по теореме 2 для групп $\mathscr{F}$ также существует инвариантная мера. Заметим, что в этих рассуждениях условие $\Gamma \neq \Gamma^{s}$ не используется. Если $\Gamma \neq \Gamma^{s}$, то и $\mathscr{F} \neq \mathscr{F}^{s}$.

В действительности мы доказали большее. Если $\widetilde{\Gamma}-$ произвольная нормальная подгруппа групшы $G$ с инвариантной мерой, то группа $F=\left\langle\widetilde{\Gamma}, H_{G}\right\rangle$ также является нормальной подгруппой с инвариантной мерой и $F^{s}=H_{G}$.

Рассмотрим множество всех нормальных подгрупп $F$ с инвариантной мерой и со свойством $F^{s}=H_{G}$. Очевидно, что это множество непусто, так как к нему принадлежит подгруппа $\mathscr{F}$. Введем отношение частичного порядка следующим образом: $F_{1} \prec F_{2}$, если $F_{1} \subseteq F_{2}$. По лемме Цорна цепь $F_{0}=\mathscr{F}$ содержится в некоторой максимальной цепи $\left\{F_{\alpha}\right\}_{\alpha \in \mathscr{A}}$. Рассмотрим объединение $M=\bigcup_{\alpha \in \mathscr{A}} F_{\alpha}$, которое также является нормальной подгруппой. Очевидно, что для подгруппы $M$ также вьполняется условие $M^{s}=H_{G}$. Так как $\mathscr{F} \neq \mathscr{F}^{s}$, то и $M \neq M^{s}$. Тогда по предложению 5 существует мера, инвариантная относительно $M$. Очевидно, что $M$ будет 0 -максимальной подгруппой. Лемма доказана.

Теперь мы можем доказать наш основной результат о существовании проективно-инвариантной меры.

Теорема С. Пусть $G \subseteq \operatorname{Homeo}(\mathbb{R})$ содержит нормальную подгруппу $\Gamma \subseteq$ Homeо $_{+}(\mathbb{R})$ с инвариантной мерой и свободно действующим әлементом. Для существования борелевской меры, конечной на компактах и проективно-инвариантной относительно группь $G$, необходимо и достаточно, чтобы факторгруппа $G / H_{G}$ не содержала свободной подгруппь с двумя образующими.

ДокАЗАТЕльСтво. Заметим, что $H_{G}=H_{G_{+}}$. По предложению 5 для группы $G$ и ее нормальной подгрупш $G_{+}$проективно-инвариантная мера либо существует, либо нет одновременно. С другой стороны, для факторгрупп $G / H_{G}, G_{+} / H_{G}$ свободные подгруппы с двумя образующими либо существуют, либо нет также одновременно. Поэтому нам достаточно доказать теорему для групп $G \subseteq \mathrm{Homeo}_{+}(\mathbb{R})$. Но для таких групп в силу леммы 3 теорема С является переформулировкой теоремы 4 .

По проективно-инвариантной мере $\mu$ мы можем определить гомоморфизм $A_{G}: G \rightarrow \mathbb{R}$, где для любого $g \in G A_{G}(g)=c(g)(c(g)$ из определения 3$)$.

Пусть $\mathscr{C}=\operatorname{ker} A_{G}, \mathscr{H}=\operatorname{ker} A_{G} \cap G^{s}$. Приведем один результат о структуре групп с проективно-инвариантной мерой [5, теорема 7.3].

Теорема 8. Пусть $G \subseteq$ Homeо+ $(\mathbb{R})$, для которой существует проективно-инвариантная мера $\mu$. Тогда $\mathscr{H} \subseteq \mathscr{C} \subseteq G, \mathscr{H}$ и $\mathscr{C}-$ нормальные подгруппь группы $G$, факторгруппа $G / \mathscr{H}$ изоморфна подгруппе группы аффинных преобразований $\mathbb{R}, a$ факторгруппы $G / \mathscr{C}, \mathscr{C} / \mathscr{H}$ изоморфны подгруппам аддитивной әруппы $\mathbb{R}$. Более mого, $\mathscr{C}^{s}=\mathscr{H}$.

Уточним структуру групп с проективно-инвариантной мерой $[5$, следствие 7.3 , леммa 7.4]. 
ПРЕДЛОЖЕНИЕ 8. Пусть $G \subseteq \mathrm{HomeO}_{+}(\mathbb{R})$, для которой существует проективно-инвариантная мера, но не существует инвариантной меры. Тогда $\mathscr{H}=G_{\infty}^{s}$, $G \backslash G^{s} \neq \varnothing u G \backslash G^{s} \subseteq \mathscr{C}$, а факторгруппа $\mathscr{C} / \mathscr{H}$ неииклическая.

Приведем один результат о связи минимальных множеств групшы и ее нормальных подгрупш [2, лемма 2.3].

ПРЕДЛОЖЕНИЕ 9. Пусть $G \subseteq \mathrm{Homeo}_{+}(\mathbb{R})$, a $Г$ - ее нормальная подгруппа. Если минимальное множество подгруппы Г непусто и недискретно, то минимальное множество группы $G$ также непусто и совпадает с минимальным множеством $\Gamma$.

Лемма 4. Пусть $G \subseteq \mathrm{HomeO}_{+}(\mathbb{R})$, для которой существует проективно-инвариантная мера $\mu$, но не существует инвариантной меры. Тогда $\mathscr{H}=H_{G} u$ $\mathscr{C}=C$.

ДокАЗАТЕЛЬСтво. По предложению $8 G \backslash G^{s} \neq \varnothing$, т.е. существует свободно действующий элемент $\widetilde{g} \in \mathscr{C}$. Тогда по теореме 7 для грушы $G$ сушествует минимальное множество. Так как элемент $\widetilde{g}$ свободно действующий, то минимальное множество неограничено ни слева, ни справа. Из условия нецикличности факторгрупшы $\mathscr{C} / \mathscr{H}$ и того, что $\mathscr{C}^{s}=\mathscr{H}$ (теорема 8), следует, что минимальное множество групшы $G$ недискретное и обозначается через $E(G)$. Из недискретности минимального множества $E(G)$, его неограниченности слева и справа и условия $\mathscr{H}=G_{\infty}^{s}$ (см. предложение 8) следует, что $H_{G} \subseteq \mathscr{H}$.

С другой стороны, так как $\tilde{g} \in \mathscr{C}$ - свободно действующий элемент, то в силу теоремы 7 минимальное множество групшы $\mathscr{C}$ также непусто. Из нецикличности факторгруппы $\mathscr{C} / \mathscr{H}$ и условия $\mathscr{C}^{s}=\mathscr{H}$ (теорема 8) следует, что такое минимальное множество недискретное и обозначается через $E(\mathscr{C})$. Тогда по предложению $9 E(G)=E(\mathscr{C})$. В силу определения подгруппа $\mathscr{C}$ является максимальной нормальной подгруппой, для которой проективно-инвариантная мера $\mu$ является инвариантной. Тогда по теореме 2 должно выполняться условие $\mathrm{Fix} \mathscr{C}^{s} \neq \varnothing$. Очевидно, что минимальное множество $E(\mathscr{C})$ должно принадлежать замкнутому инвариантному относительно $\mathscr{C}$ множеству Fix $\mathscr{C}^{s}$. B таком случае должно вьполняться включение $\mathscr{C}^{s} \subseteq H_{G}$. Так как $\mathscr{C}^{s}=\mathscr{H}$, то это влечет за собой включение $\mathscr{H} \subseteq H_{G}$. Сравнивая с полученньп ранее обратным включением, получим, что $H_{G}=\mathscr{H}$.

Из условий $G \backslash G^{s} \subseteq \mathscr{C}$ и $\mathscr{H}=G_{\infty}^{s}$ (предложение 8) следует включение $C \subseteq \mathscr{C}$. Так как мера $\mu$ инвариантна относительно группы $\mathscr{C}$ и существует свободно действующий элемент $\widetilde{g} \in \mathscr{C}$, то верно и обратное включение $\mathscr{C} \subseteq C$. Лемма доказана.

Лемма 5. Пусть $G \subseteq \mathrm{Homeo}_{+}(\mathbb{R})$ содержит нормальную подгруппу $\Gamma$ с инвариантной мерой и свободно действующим әлементом. Тогда факторгруппа $G / H_{G}$ либо содержит свободную подгруппу с двумя образующими, либо является разреиимой ступени не более двух.

ДокАЗАТЕЛЬСТво. Пусть $G / H_{G}$ не содержит свободной подгрупшы с двумя образуюшими. По теореме С для групшы $G$ существует проективно-инвариантная мера. Если для групшы $G$ сушествует инвариантная мера, то по теореме 2 множество Fix $G^{s}$ непусто. Из непустоты множества Fix $G^{s}$ следует, что множество $G^{s}$ образует групу, т.е. 
$\left\langle G^{s}\right\rangle=G^{s}$. В силу инвариантности множества Fix $G^{s}$ минимальное множество будет принадлежать $\mathrm{Fix} G^{s}$. Если минимальное множество дискретное, то по определению $H_{G}=G^{s}$. Если минимальное множество недискретное, то в силу определения множества $\mathrm{Fix} G^{s}$ также справедливо условие $H_{G}=G^{s}$. В таком случае коммутативность факторгруппы $G /\left\langle H_{G}\right\rangle$ будет следовать из теоремы 6 .

Если для группы $G$ не существует инвариантной меры, то по лемме $4 H_{G}=\mathscr{H}$. Тогда по теореме 8 факторгруппа $G / H_{G}$ является разрешимой ступени два.

Обратное утверждение очевидно.

TЕОрема $\mathrm{C}^{\prime}$. Пусть $G \subseteq \operatorname{Homeo}(\mathbb{R})$ содерәит нормальную подгруппу $\Gamma \subseteq$ Homeо+ $_{+}(\mathbb{R})$ с инвариантной мерой и свободно действуюшим әлементом. Тогда факторгруппа $G / H_{G}$ либо содержит свободную подгруппу с двумя образующими, либо является разрешимой ступени не более трех (содержит разрешимую подгруппу ступени и индекса не более двух).

ДокАЗАтЕЛьСтво. Заметим, что $H_{G}=H_{G_{+}}$. Для факторгрупш $G / H_{G}, G_{+} / H_{G}$ свободные подгрупшы с двумя образуюшими либо существуют, либо нет одновременно. Если факторгруппа $G_{+} / H_{G}$ не содержит свободной подгруппы с двумя образующими, то по лемме 5 она является разрешимой ступени не более двух. В таком случае факторгруппа $G / H_{G}$ будет содержать разрешимую группу ступени и индекса не более двух.

Автор признателен участникам семинара “Динамические системы и эргодическая теория" и ее руководителям Д. В. Аносову, А. М. Степину и особенно Р. И. Григорчуку за полезные обсуждения и внимание к работе.

\section{СПИСОК ЦИТИРОВАННОЙ ЛИТЕРАТУРЫ}

[1] Margulis G. Free subgroups of the homeomorphism group of the circle // C. R. Acad. Sci. Paris. Ser. I. 2000. V. 331. P. 669-674.

[2] Бекларян Л. А. К вопросу о классификации групп гомеоморфизмов $\mathbb{R}$, сохраняющих ориентацию. III. $\omega$-проективно-инвариантные меры // Матем. сб. 1999. Т. 190. № 4. С. 43-62.

[3] Солодов В. В. Гомеоморфизмы окружности и слоения // Изв. АН СССР. Сер. матем. 1984. Т. 48. № 3. С. 599-613.

[4] Солодов В.В. Гомеоморфизмы прямой и слоения // Изв. АН СССР. Сер. матем. 1982. Т. 46. № 5. C. 1047-1060.

[5] Бекларян Л. А. К вопросу о классификации групп гомеоморфизмов $\mathbb{R}$, сохраняющих ориентацию. II. Проективно-инвариантные меры // Матем. сб. 1996. Т. 187. № 4. С. 3-28.

[6] Бекларян Л. А. К вопросу о классификации групп гомеоморфизмов $\mathbb{R}$, сохраняющих ориентацию. І. Инвариантные меры // Матем. сб. 1996. Т. 187. № 3. С. 23-54.

[7] Бекларян Л. А. Структура факторгруппы группы гомеоморфизмов $\mathbb{R}$, сохраняющих ориентацию, по подгруппе, порожденной объединением стабилизаторов // Докл. РАН. 1993. Т. 331. №2. C. $137-139$. 